\title{
The OUT OF Africa Hypothesis of Comparative ECONOMiC DeVElopMent: COMMON MISCONCEPTIONS
}

\author{
QUAMrUl H. ASHRAF, OdED GALOR, AND MARC KLEMP*
}

\begin{abstract}
The importance of the prehistoric migration of anatomically modern humans from Africa for comparative economic development has been the focus of a vibrant research agenda in the past decade. This influential literature has attracted the attention of some scholars from other disciplines, and in light of existing methodological gaps across fields, has perhaps unsurprisingly generated some significant misconceptions. This article examines the critical views expressed by some scholars from other disciplines, and establishes that they are based on fundamental misunderstandings of the statistical methodology, the conceptual framework, and the scope of the analysis that characterize this influential literature.
\end{abstract}

JEL classification codes: O11, N10, N30, Z10

Keywords: Comparative development, interpersonal population diversity, the out of Africa hypothesis

*Ashraf: Department of Economics, Williams College (email: Quamrul.H.Ashraf@williams.edu); Galor: Brown University, NBER, CEPR, IZA and CeSifo (email: Oded_Galor@ brown.edu); Klemp: University of Copenhagen and CEPR (email: Marc.Klemp@econ.ku.dk). 


\section{Introduction}

The past two decades have witnessed the emergence of a large and influential body of research that has focused on uncovering the roots of comparative economic development across regions, countries, and ethnic groups. A significant portion of this line of inquiry has explored the role of the prehistoric migration of anatomically modern humans from Africa in the differential development patterns across the globe.

This line of research has explored the persistent effect of the prehistoric exodus of Homo sapiens from Africa on comparative economic development across societies from the dawn of civilization to the contemporary era. In particular, this research suggests that greater migratory distances from the cradle of mankind in East Africa to the indigenous settlements of the ancestral populations of nations or ethnic groups diminished their levels of interpersonal diversity and, thereby, generated a persistent hump-shaped influence on development outcomes, reflecting a fundamental trade-off between beneficial and detrimental effects of diversity on productivity at the societal level (Ashraf and Galor, 2013a, 2018; Ashraf, Galor, and Klemp, 2018a). Although diversity may reduce interpersonal trust, cooperation, and social cohesiveness, and can thus adversely affect the productivity of society, complementarity across diverse productive traits may stimulate innovations and gains from specialization, and can thereby contribute to society's economic performance. Therefore, in the presence of diminishing marginal effects of diversity and homogeneity on productivity, the aggregate economic performance of ethnic groups, countries, or regions that are characterized by intermediate levels of diversity would be expected to be higher than that associated with excessively homogenous or heterogeneous societies.

Consistent with each of the fundamental building blocks of this hypothesis, interpersonal diversity, as proxied by diversity in genetic markers, appears to have contributed to ethnic and cultural heterogeneity (Ashraf and Galor, 2013b), diminished interpersonal trust (Ashraf and Galor 2013a) and the emergence of civil conflicts (Arbatli, Ashraf, Galor, and Klemp, 2019). Moreover, evidence suggests that interpersonal diversity may foster innovative activity (Ashraf and Galor, 2013a; Cook and Fletcher, 2018), occupational heterogeneity, and gains from specialization (Depetris-Chauvin and Özak, 2018). Further, interpersonal diversity may have shaped the nature of both precolonial and contemporary political institutions. In particular, although diversity may have triggered the development of institutions for mitigating the adverse influence of population diversity on social cohesion, the contribution of diversity to economic inequality and class stratification may have ultimately led to the formation and persistence of extractive and autocratic institutions (Galor and Klemp, 2018).

The literature on the influence of interpersonal diversity on comparative development across societies has attracted the attention of the scholarly community beyond the discipline of economics and, given methodological divisions, perhaps unsurprisingly generated unfounded criticisms. This article examines these criticisms and demonstrates that they are based on fundamental misconceptions regarding the conceptual framework, the statistical methodology, and the scope of the analysis. 


\section{Common Misconceptions}

Three main criticisms have been raised by scholars from other disciplines; predominantly, cultural anthropologists. As established below, each of them is rooted in a basic misunderstanding of the conceptual framework, the statistical methodology, the scope of the analysis, and its policy implications. ${ }^{1}$

\subsection{Potential Under-Estimation of Population Density in Pre-Columbian America}

\section{Misconception I}

The population data employed in the analysis of development outcomes in the pre-colonial period are imperfectly measured, and in particular, the population levels of precolonial Amerindian societies are underestimated, contributing to the hypothesized hump-shaped relationship.

\section{Response}

The historical analysis of Ashraf and Galor (2013a) accounts for the possibility that the data on population density prior to the year 1500 could be afflicted by measurement errors, demonstrating that this issue has no bearing on the validity of their empirical findings.

a. Since population density is the dependent variable, classical measurement error in this variable does not generate bias in the estimates of the hump-shaped influence of diversity on historical development. In fact, in the absence of classical measurement error, the statistical significance of the estimates would be even higher.

b. If there are systematic differences across continents in the measurement of historical population density (e.g., if historical population density in the Americas is indeed consistently underestimated), then the estimated effects could have been biased. However, the use of continent fixed effects in the statistical analysis of Ashraf and Galor (2013a), assures that the influence of diversity on historical development is identified based on intersocietal variations within continents, rather than across continents, and hence systematic under-estimation of the population levels in the Americas would have no bearing on the results.

c. Employing an alternative measure of historical development based upon the extent of urbanization, rather than population density in 1500 (Ashraf and Galor, 2011), does not qualitatively alter the hump-shaped influence of interpersonal diversity on historical development, as depicted in Figure $1 .^{2}$

\footnotetext{
${ }^{1}$ In addition to the three misconceptions discussed below, Rosenberg and Kang (2015) suggest that the hump-shaped effect population diversity on population density in the year 1500 is not robust if one uses the extended sample of Pemberton et al. (2013). As elaborated in the formal response of Ashraf, Galor, and Klemp (2018b), this assertion is based on elementary statistical errors. In fact, as established in Ashraf, Galor, and Klemp (2018a), and as depicted partly in Figure 2, the hump-shaped effect of interpersonal population diversity on population density is even stronger when using the extended sample of Pemberton et al. (2013), and the highly significant hump-shaped impact population diversity is present over the entire pre-colonial period for which population data is available (i.e., the 10,000BCE to 1500CE timeframe).

${ }^{2}$ It should be noted that the data source for urbanization rates in 1500 is independent of the source for historical population density.
} 
Panel A:

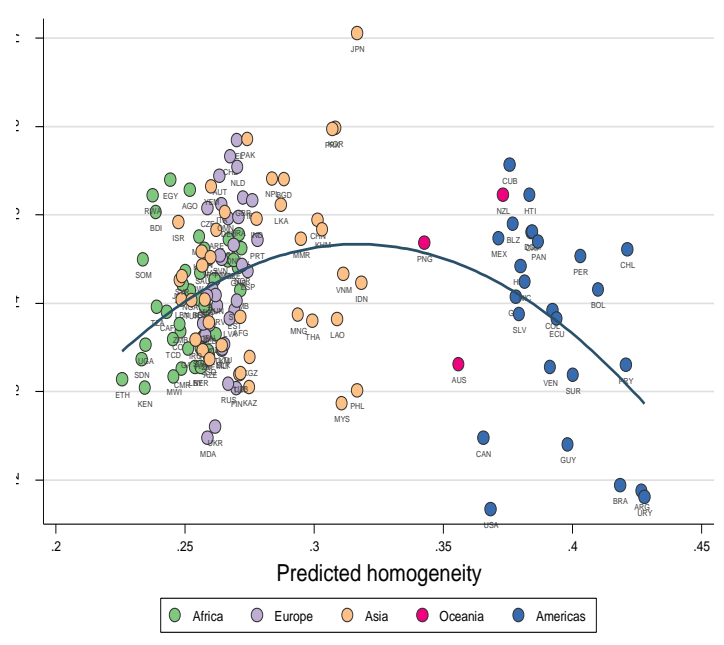

Panel B:

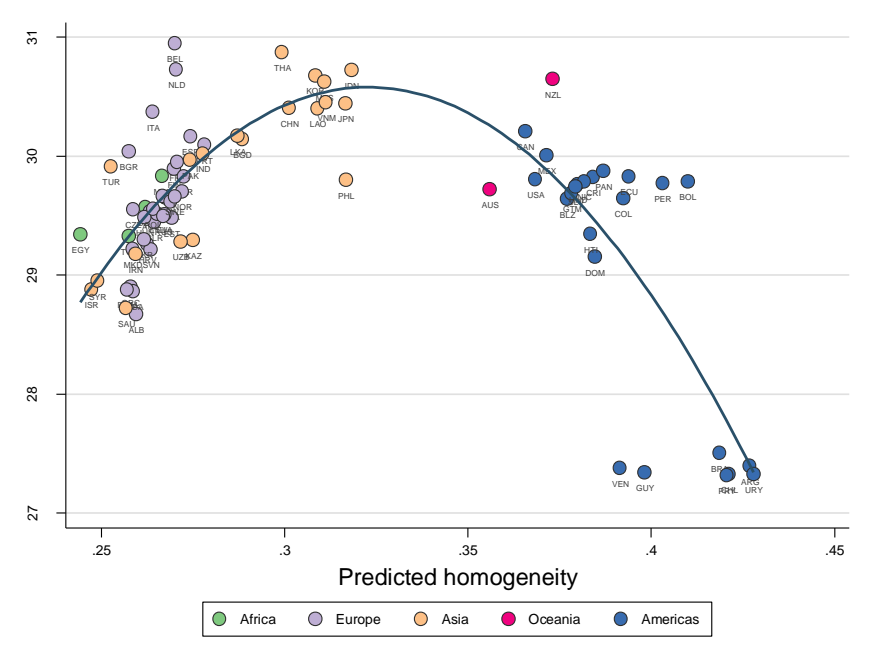

Figure 1. Interpersonal Diversity and Historical Comparative Development across Countries

Notes: This figure depicts the cross-country hump-shaped influence of predicted homogeneity (i.e., 1 minus interpersonal diversity predicted by migratory distance from East Africa) on economic development in the year 1500, as reflected by either log population density (Panel A) or log urbanization rate (Panel B), conditional on the timing of the Neolithic Revolution, land productivity, and continent/regional fixed effects. Source: Ashraf and Galor (2013a).

d. The hump-shaped impact of interpersonal population diversity on historical population densities over the period 10,000BCE - 1500CE is established at the ethnic level (across the extended sample of ethnic groups in Pemberton et al., 2013). As reported in Ashraf, Galor and Klemp (2018a), the impact is highly significant for each thousand-year interval over this time period, as depicted partly in Figure 2.

e. The main analysis of Ashraf and Galor (2013a) is based on contemporary comparative economic development, in which the dependent variable is income per capita in the year 2000 (rather than historical population density), and the robust hump-shaped impact of interpersonal population diversity is fully evident, as depicted in Figure 3A.

f. As established by Ashraf, Galor and Klemp (2018a) and depicted in Figure 3B, the humpshaped impact of interpersonal population diversity is robust to the use of an alternative measure of contemporary economic development, based on luminosity per capita across the extended sample of ethnic groups in Pemberton et al. (2013). 


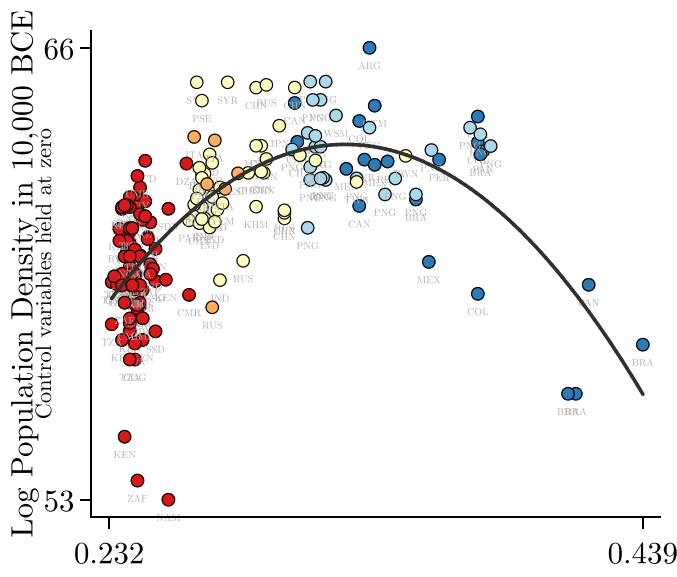

Population Homogeneity (Instrumented)

- Americas $\bigcirc$ Oceania $\bigcirc$ Asia $\bigcirc$ Europe $\bigcirc$ Africa

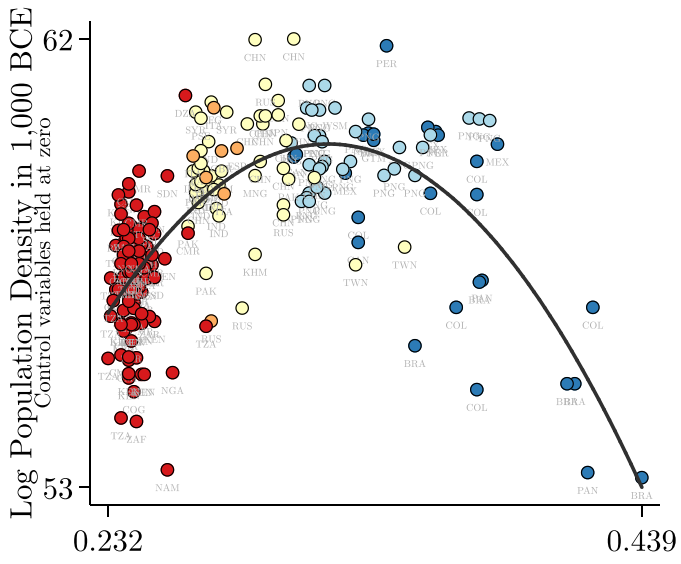

Population Homogeneity (Instrumented)

- Americas $\bigcirc$ Oceania $\bigcirc$ Asia $\bigcirc$ Europe $\bigcirc$ Africa
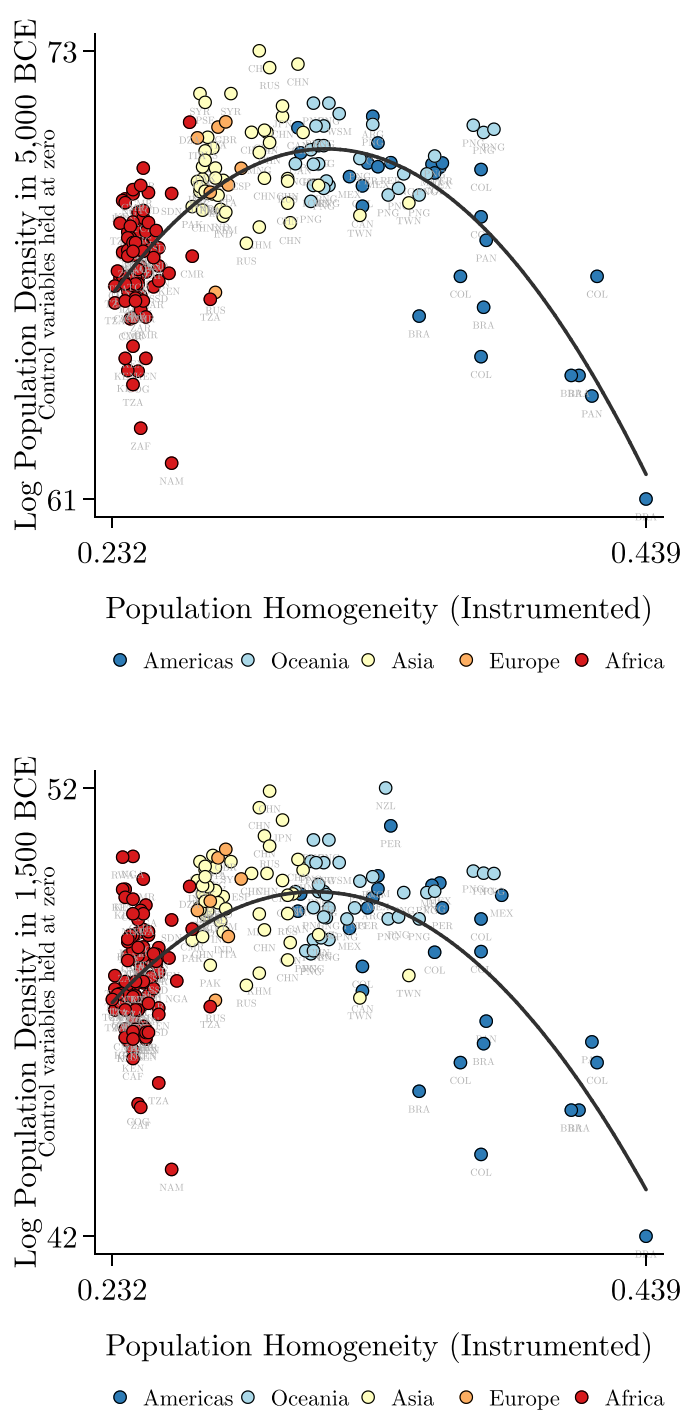

Figure 2. Interpersonal Diversity and Population Density across Ethnic Groups over the Period 10,000BCE - 1500CE

Notes: This figure depicts the hump-shaped influence of interpersonal diversity on population density across ethnic groups in the extended sample of Pemberton et al. (2013). It depicts the impact of observed genetic homogeneity (i.e., 1 minus observed diversity), instrumented by the migratory distance from East Africa, on population density in the years 10,000BCE, 5,000BCE, 1000BCE, and 1500CE, conditional geographical controls and regional fixed effects. Source: Ashraf, Galor, and Klemp (2018a). 


\section{Panel A:}

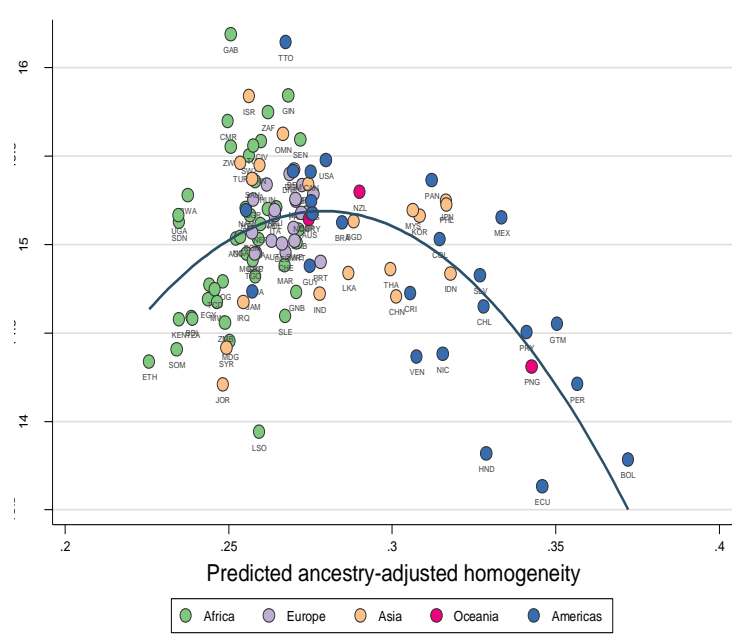

Panel B:

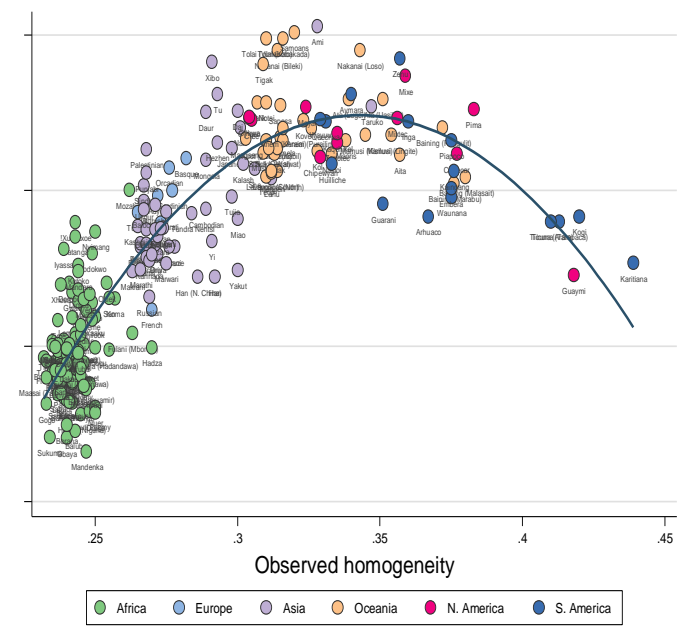

Figure 3. Interpersonal Diversity and Contemporary Comparative Development across Countries and Ethnic Groups

Notes: This figure depicts the hump-shaped influence of interpersonal diversity on contemporary economic development across countries and ethnic groups. Panel A depicts the relationship across countries between predicted ancestry-adjusted homogeneity (i.e., 1 minus interpersonal diversity predicted by the migratory distances of a country's ancestral populations from East Africa and the pairwise migratory distances among them) and log income per capita in the year 2000, conditional on the ancestry-adjusted timing of the Neolithic Revolution, land productivity, a vector of institutional, cultural, and geographical determinants of development, and continent/regional fixed effects. Panel B depicts the relationship across ethnic groups between observed homogeneity (i.e., 1 minus expected heterozygosity) and log average light intensity per capita in the 1992-2013-time horizon, conditional on absolute latitude, soil quality, type of landmass, group size, institutional characteristics (extent of jurisdictional hierarchy and type of class stratification), and regional fixed effects. Sources: Ashraf and Galor (2013a) and Ashraf, Galor, and Klemp (2018a).

Hence, concerns about the potential underestimation of populations in the Americas in the preColumbian era and its impact on the analysis are based on a fundamental misunderstanding of the statistical methodology employed in the research of Ashraf and Galor (2013a). Moreover, the presence of a hump-shaped impact of interpersonal population diversity on: (i) the rates of urbanization in the pre-Columbian period, (ii) the population density of ethnic groups over the period 10,000BCE-1500CE, and (iii) contemporary measures of income per capita (for which potential mismeasurements of population density in the pre-Columbian period are entirely irrelevant), further highlight the nature of this uninformed criticism.

\subsection{The Mapping from Diversity in Neutral Genetic Markers to Social Outcomes}

\section{Misconception II}

Expected heterozygosity in neutral genetic markers, employed to proxy for the degree of interpersonal diversity within ethnic groups, does not reflect diversity in functional (phenotypic) markers and, therefore, cannot influence behavioral and social interactions. 


\section{Response}

The measures of observed genetic diversity for the 53 ethnic groups in the Human Genome Diversity Project (HGDP-CEPH), as well as in the more recently extended data set of Pemberton et al. (2013) are indeed based neutral genetic markers and, thus, do not directly reflect diversity in functional (phenotypic) markers.

However, the core analysis of Ashraf and Galor (2013a) is based not on observed genetic diversity but rather on predicted diversity as implied by the migratory distance from Africa to the ancestral populations of contemporary nations. In particular, in order to overcome sample limitations and potential concerns about reverse causality associated with the use of observed genetic diversity, Ashraf and Galor (2013a) exploit the pronounced impact of migratory distance from East Africa on observed genetic diversity across ethnic groups in the HGDP-CEPH sample (as depicted in Figure 4) in order predict interpersonal diversity for all societies, based on the geographical locations of their ancestral populations in the year 1500, relative to East Africa.

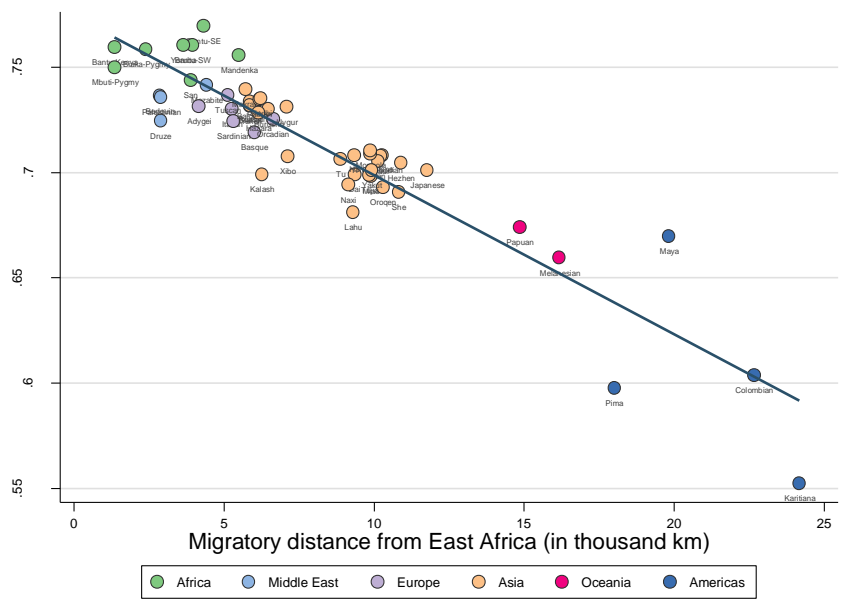

Figure 4. Expected Heterozygosity and Migratory Distance from East Africa

Notes: This figure depicts the negative influence of migratory distance from East Africa on expected heterozygosity (genetic diversity) across the 53 ethnic groups that constitute the HGDP-CEPH Human Genome Diversity Cell Line Panel. Source: Ashraf and Galor (2013a).

Importantly, since migratory distance from East Africa has a negative influence on various forms of intragroup phenotypic diversity, predicted interpersonal diversity is a valid proxy for diversity in phenotypically and behaviorally expressed traits. In particular, mounting evidence in the fields of physical and cognitive anthropology suggest that an ancient serial founder effect originating in East Africa affected observed worldwide patterns in various forms of intragroup morphological and cognitive diversity, including interpersonal diversity in skeletal features pertaining to cranial characteristics (Manica et al. 2007; von Cramon-Taubadel and Lycett 2008; Betti et al. 2009), dental attributes (Hanihara 2008), pelvic traits (Betti et al. 2013), and birth canal morphology (Betti and Manica, 2018), as well as intralingual phonemic diversity (Atkinson 2011). 
Hence, the assertion that inferences on the impact of interpersonal diversity on socio-economic outcome cannot be made, because the measure of genetic diversity in the HGDP-CEPH sample is based on neutral genetic markers, yet again reflects a fundamental misunderstanding of the empirical strategy of Ashraf and Galor (2013a). Since migratory distance from Africa affects diversity in neutral genetic markers as well as diversity in phenotypically expressed morphological and cognitive traits, the empirical strategy used by Ashraf and Galor (2013a), based upon predicted diversity, is well positioned to capture the effect of interpersonal population diversity on socioeconomic outcomes.

\subsection{Policy Implications}

\section{Misconception III}

Ashraf and Galor's (2013a) assertion that there is a productivity-maximizing level of diversity could be used to justify disturbing policy prescriptions, such as the forcible movement or "engineering" of populations, designed to achieve an "optimal" diversity level in a population.

\section{Response}

The view of uninformed critics that the finding that intermediate levels of interpersonal diversity in heritable traits tend to be most conducive to economic development can be used to justify disturbing policy prescriptions reflects a basic misunderstanding of the most fundamental insight of this research agenda and its main policy implications:

a. The importance of diversity for economic prosperity has increased over the course of human history, and unlike the historical (i.e., pre-colonial) world, the most productive nations tend to be more diverse.

b. Education policy geared towards respect for pluralism and, thus, towards mitigating the cost of diversity could further increase the importance of diversity for economic prosperity.

Ashraf and Galor (2013a) and Ashraf, Galor, and Klemp (2018a) document a fundamental tradeoff associated with the influence of interpersonal diversity, as reflected by predicted diversity, on economic performance. Nevertheless, the finding that predicted diversity, based on migratory distance from Africa, has been a deep determinant of economic development does not imply that the composition of heritable traits in a population governs its economic destiny. Migratory distance from Africa affected interpersonal diversity via the interaction of biological, geographical, and cultural attributes and policies could be aimed at conditioning these intervening channels.

In particular, the influence of diversity on productivity implies that a society can shape the context in which the existing diversity of its population influences socioeconomic outcomes, by enacting policies to harness the beneficial effects of the existing level of diversity and mitigate its potentially detrimental consequences. Overly diverse societies could focus on fostering interpersonal trust and mediating the potential for social conflict, by encouraging civic participation, improving the quality of political institutions, and mitigating inefficiencies and distortions in the provision of public goods. Overly homogenous societies, on the other hand, could aim to increase diversity in skills, occupations, and training programs in order to foster specialization and innovative activity. In both cases, the orientation of the educational system appears to be the most promising avenue: 
education can help instill the values of tolerance needed in overly diverse societies, and it can promote cultural receptiveness to different types of productivity-enhancing knowledge that may be lacking in overly homogenous societies.

\section{Concluding Remarks}

Ashraf and Galor (2013a) advanced the hypothesis that migratory distances from the cradle of mankind in East Africa to the indigenous settlements of the ancestral populations of nations or ethnic groups diminished their levels of interpersonal diversity and, thereby, generated a persistent hump-shaped influence on development outcomes, reflecting a fundamental trade-off between beneficial and detrimental effects of diversity on productivity at the societal level. Although diversity may reduce interpersonal trust, cooperation, and social cohesiveness, and can thus adversely affect the productivity of society, complementarity across diverse productive traits may stimulate innovations and gains from specialization, and can thereby contribute to society's economic performance.

The literature on the influence of interpersonal diversity on comparative development across societies has attracted the attention of the scholarly community beyond the discipline of economics and, given methodological divisions, has perhaps unsurprisingly generated unfounded criticisms. This article examines the main criticisms of some scholars in other disciplines and demonstrates that they are based on fundamental misunderstandings of the statistical methodology employed by Ashraf and Galor, their conceptual framework, and the scope of their analysis. 


\section{REFERENCES}

Arbatl, Cemal Eren, Quamrul H. Ashraf, Oded Galor, and Marc Klemp. 2019. “Diversity and Conflict." Econometrica (forthcoming).

Ashraf, Quamrul, and Oded Galor. 2011. "Dynamics and Stagnation in the Malthusian Epoch." American Economic Review, 101(5): 2003-2041.

Ashraf, Quamrul, and Oded Galor. 2013a. "The 'Out of Africa' Hypothesis, Human Genetic Diversity, and Comparative Economic Development." American Economic Review, 103(1): 146.

Ashraf, Quamrul, and Oded Galor. 2013b. "Genetic Diversity and the Origins of Cultural Fragmentation," American Economic Review, 103(3): 528-533.

Ashraf, Quamrul H., and Oded Galor. 2018. "The Macrogenoeconomics of Comparative Development," Journal of Economic Literature, 56(3): 1119-1155.

Ashraf, Quamrul H., Oded Galor, and Marc Klemp. 2018a. "Population Diversity and Differential Paths of Long-Run Development since the Neolithic Revolution." Unpublished.

Ashraf, Quamrul, Oded Galor, and Marc Klemp. 2018b. "Interpersonal Diversity and Societally Important Disparities across Populations: A Reply to Rosenberg and Kang." Unpublished.

Atkinson, Quentin D. 2011. "Phonemic Diversity Supports a Serial Founder Effect Model of Language Expansion from Africa." Science, 332(6027): 346-349.

Betti, Lia, François Balloux, William Amos, Tsunehiko Hanihara, and Andrea Manica. 2009. "Distance from Africa, Not Climate, Explains Within-Population Phenotypic Diversity in Humans." Proceedings of the Royal Society B: Biological Sciences, 276(1658): 809-814.

Betti, Lia, Noreen von Cramon-Taubadel, Andrea Manica, and Stephen J. Lycett. 2013. "Global Geometric Morphometric Analyses of the Human Pelvis Reveal Substantial Neutral Population History Effects, Even across Sexes.” PLoS ONE, 8(2): e55909.

Betti, Lia, and Andrea Manica. 2018. "Human Variation in the Shape of the Birth Canal is Significant and Geographically Structured." Proceedings of the Royal Society B: Biological Sciences, 285(1889), DOI: 10.1098/rspb.2018.1807.

Cook, C. Justin, and Jason M. Fletcher. 2018. "High School Genetic Diversity and Later-life Student Outcomes: Micro-level Evidence from the Wisconsin Longitudinal Study." Journal of Economic Growth, 23(3): 307-339.

Depetris-Chauvin, Emilio, and Ömer Özak. 2018. "The Origins of the Division of Labor in PreModern Times.” IZA Discussion Paper 11554, Institute for the Study of Labor (IZA).

Galor, Oded, and Marc Klemp. 2018. "The Roots of Autocracy." National Bureau of Economic Research Working Paper 23301.

Hanihara, Tsunehiko. 2008. "Morphological Variation of Major Human Populations Based on Nonmetric Dental Traits.” American Journal of Physical Anthropology, 136(2): 169-182.

Manica, Andrea, William Amos, François Balloux, and Tsunehiko Hanihara. 2007. "The Effect of Ancient Population Bottlenecks on Human Phenotypic Variation." Nature, 448(7151): 346-348. 
Pemberton, Trevor J., Michael DeGiorgio, and Noah A. Rosenberg. 2013. "Population Structure in a Comprehensive Genomic Data Set on Human Microsatellite Variation." G3: Genes, Genomes, Genetics, 3(5): 891-907.

Prugnolle, Franck, Andrea Manica, and François Balloux. 2005. "Geography Predicts Neutral Genetic Diversity of Human Populations." Current Biology, 15(5): R159-R160.

Rosenberg, Noah A., and Jonathan T. L. Kang. 2015. "Genetic Diversity and Societally Important Disparities." Genetics, 201(1): 1-12. 